\title{
„Im Schatten der Hierarchie“ - Eine empirische Annäherung an das Führungsverständnis und Orientierungen des Führungshandelns in Weiterbildungsorganisationen
}

\author{
Dörthe Herbrechter (D)
}

Eingegangen: 17. Mai 2016 / Angenommen: 8. Juli 2016 / Online publiziert: 10. August 2016 (C) Der/die Autor(en) 2016. Dieser Artikel ist eine Open-Access-Publikation.

Zusammenfassung Mit dem Managementkonzept wurde und wird in der Weiterbildungsforschung bislang vor allem eine primär auf Strukturen und Sachprozesse fokussierte Steuerung in den Blick genommen. An diesen bisherigen Forschungsschwerpunkt anknüpfend, rückt der vorliegende Beitrag nun Führung, d. h. die Koordination der Organisationsmitglieder durch eine gezielte Einflussnahme der Vorgesetzten, in den Mittelpunkt des Interesses. Auf der Grundlage eines kontrastiven Fallvergleichs wird untersucht, wie Leitungspersonen durch ihr Führungsverständnis bzw. ihre damit korrespondierenden Handlungsorientierungen hierarchische und nicht-hierarchische Formen der Handlungskoordination begünstigen und inwiefern hierbei organisationale Rahmenbedingungen eine Rolle spielen.

Schlüsselwörter Führung · Organisationsinterne Handlungskoordination · Hierarchie · Organisationsstrukturelle Bedingungen

\section{"In the shadow of hierarchy" - An empirical approach to leadership un- derstanding and orientations of action in organisations of continuing ed- ucation}

\begin{abstract}
So far in the field of continuing education research the management concept is and has been primarily focused. Therefore the research is still centred on directing structures and objective processes. Thus, this article concentrates on leadership, i. e. the superior's intended influence on the coordination of organisation
\end{abstract}

In der deutschsprachigen Literatur zur Governance-Perspektive ist die Vorstellung vom „Schatten der Hierarchie“ maßgeblich von Mayntz und Scharpf geprägt worden (u. a. Mayntz und Scharpf 1995; Scharpf 1991).

D. Herbrechter $(\bowtie)$

Deutsches Institut für Erwachsenenbildung, Leibniz-Zentrum für Lebenslanges Lernen e. V. (DIE),

Bonn, Deutschland

E-Mail: herbrechter@die-bonn.de 
members. Based on a contrastive case comparison, it analyses the enhancement of hierarchical and non-hierarchical forms of action coordination triggered by the superior's leadership understanding and orientations of action. It also analyses the role of organisational framework in this context.

Keywords Leadership - Coordination of action in organisations · Hierarchy · Structural conditions

\section{Einleitung}

Wie werden Organisationen der Weiterbildung geleitet? Auch wenn diese Frage trivial erscheinen mag, lässt sie sich doch nicht ohne weiteres beantworten. Denn bislang liegen in der nationalen und internationalen Weiterbildungsforschung nur wenige Studien vor, die sich mit der Leitung von Weiterbildungsorganisationen als Hauptuntersuchungsgegenstand beschäftigen (vgl. Robak 2012, S. 505).

Welche empirischen Befunde in den jeweiligen Studien diskutiert werden (können), hängt auch davon ab, inwieweit sich die Forschung an theoretischen Modellen der Leitung, des Managements oder der Führung orientiert. Während sich für die Berufsrollenbezeichnungen der Praxis der Begriff „Leiter“ bzw. „Leiterin“ durchgesetzt zu haben scheint (vgl. z. B. Kade et al. 2007, S. 144; Tietgens 1994), findet in der Weiterbildungsforschung häufiger der Begriff „Management“ Berücksichtigung (vgl. Robak 2015, S. 124).

Dabei zeigen die bisherigen Forschungsergebnisse, dass das Managementhandeln in Weiterbildungsorganisationen nicht allein betriebswirtschaftlichen Effektivitätsund Effizienzvorstellungen folgt, sondern maßgeblich durch das jeweilige Bildungsverständnis und die organisationalen Rahmenbedingungen bestimmt ist (vgl. Robak 2004; vgl. auch Uhmann 2011). Sowohl in der außerbetrieblichen als auch in der innerbetrieblichen Weiterbildung scheinen unterschiedliche Managementtypen mit Merkmalen der Weiterbildungsorganisation (vgl. Robak 2004, S. 340) bzw. des Unternehmens in Verbindung zu stehen. So konnte Bäumer (1999) auch für das spezifische Segment der betrieblichen Weiterbildung zeigen, dass sich anstelle eines einheitlichen Typs vielfältige Managementtypen realisieren, die je nach Branche, Betriebsgröße und weiteren Organisationseigenschaften mal mehr, mal minder erfolgversprechend zu sein scheinen. Abgesehen von diesen organisationalen Merkmalen weisen die bisherigen Forschungsergebnisse zudem auf personale Merkmale hin, da sich Unterschiede des Managementverständnisses und -handelns auch auf soziale Geschlechterrollen und biografische Erfahrungen der Leitungspersonen zurückführen lassen (vgl. Sauer-Schiffer 2000; zur geschlechtlichen Rahmung von Leitung s. auch Schlüter 2012).

Die Weiterbildungsforschung hat also bereits erste Erkenntnisse über das Management von Weiterbildungsorganisationen gewonnen. Bislang enthalten die vorliegenden Untersuchungen allerdings nur vereinzelt Hinweise auf leadership bzw. Führung. Analytisch gerät dabei jedoch jeweils Unterschiedliches in den Fokus: Von Management ist für gewöhnlich entweder zur Differenzierung verschiedener Hierarchieebenen (z. B. Top-Management, mittleres Management etc.) oder für die Be- 
trachtung einer primär auf Strukturen und Sachprozesse bezogenen Koordination die Rede. Mit dem Begriff „Führung“ richtet sich das Hauptaugenmerk demgegenüber eher auf die Gestaltung von Interaktionszusammenhängen, d. h. auf die Koordination der Mitarbeitenden durch eine gezielte Einflussnahme der Vorgesetzten (vgl. Neuberger 2002, S. 48-50; Weinert 2004, S. 459).

Vor diesem Hintergrund rückt der vorliegende Beitrag „Führung“ in den Mittelpunkt des Interesses und knüpft damit an ein Konzept an, das in der internationalen Diskussion unter dem Stichwort educational leadership bereits seit längerem und vor allem in der angloamerikanischen Literatur Aufmerksamkeit erfährt (vgl. z. B. Preedy et al. 2012).

Die vorwiegend psychologisch geprägte Führungsforschung betrachtet Führung in Organisationen primär als vertikale Einflussnahme einer/s Vorgesetzten auf ihr/ihm unterstellte Mitarbeitende. Für das Bestehen und Funktionieren von Organisationen ist diese Form der hierarchischen Führung insofern besonders bedeutsam, als Vorgesetzte durch ihr Führungsverständnis und -handeln der organisationsstrukturell fixierten Stellen-, Aufgaben- und Zielhierarchie in konkreten Interaktionszusammenhängen Geltung verleihen und so dazu beitragen, die arbeitsteilig erbrachten Handlungsbeiträge mit Blick auf die Organisationsziele zu koordinieren (vgl. Rosenstiel et al. 2005, S. 311, 313; Kieser und Walgenbach 2010). Hierfür werden ihnen qua Position besondere Einflusspotenziale (z. B. Privilegien der Informationssteuerung, der Weisungs-, Kontroll- und Entscheidungsbefugnis) zugestanden, so dass sie im Vergleich zu anderen Organisationsmitgliedern über privilegierte Chancen der Einflussnahme verfügen (vgl. Etzioni 1975). Mit der (Organisational) Governanceforschung lässt sich nun annehmen, dass (formale) Führungskräfte durch ihr Führungsverständnis und -handeln nicht nur die für Organisationen charakteristische Koordinationsform der Hierarchie befördern, sondern dass sie im Schatten der Hierarchie weitere Formen der Handlungsabstimmung unterstützen, wie beispielsweise Gemeinschaften, soziale Netzwerke, Wettbewerb oder Märkte (vgl. Schneider 2004, S. 178; Benz und Dose 2010a, S. 26, 31).

Vor diesem Hintergrund geht der Beitrag folgenden Forschungsfragen nach ${ }^{1}$ :

- Was verstehen Leitungspersonen unter Führung und auf welche damit verbundenen Handlungsorientierungen verweisen sie insbesondere mit Blick auf ein koordiniertes Zusammenwirken der Mitarbeitenden?

\footnotetext{
1 Mit den drei Forschungsfragen verbindet sich nicht der Anspruch, empirisch geprüfte Aussagen über die Wirksamkeit des Führungshandelns zu treffen. Da die Führungspraxis in Weiterbildungsorganisationen bislang kaum erforscht ist, sollen auf der Grundlage von Organisationsdaten und strukturierten Leitfadeninterviews die subjektiven Relevanzsetzungen und Beobachtungen der Leitungspersonen als Schlüsselpersonen für die Ausgestaltung der Organisation untersucht werden (vgl. Kieser und Walgenbach 2010, S. 167; Froschauer und Lueger 2003, S. 16-17). Wenn im Folgenden also Annahmen über Zusammenhänge zwischen Codes entfaltet werden, so geschieht dies stets im Sinne des Theorieverständnisses der Grounded Theory nach Strauss und Corbin, demgemäß entwickelte Codes systematisch mit anderen Codes in Beziehung gesetzt werden sollen. Dabei sollen keine auf ein empirisches Kollektiv verallgemeinerbaren Wirkungsaussagen getroffen, sondern ,bereichsbezogene“, theoriebildende Annahmen generiert werden, die Perspektiven für weitere Forschung aufzeigen (vgl. Böhm et al. 2008, S. 32; Strauss und Corbin 1996, S. 146, vgl. auch Herbrechter 2016).
} 
- (Wie) Befördern Leitungspersonen durch ihr Führungsverständnis und ihre entsprechenden Handlungsorientierungen die für Organisationen als grundlegend angenommene Koordinationsform der Hierarchie und inwiefern begünstigen sie im Schatten der Hierarchie weitere Koordinationsformen?

- Lassen sich die je identifizierten Ausprägungen des Führungsverständnisses und der individuellen Handlungsorientierung mit Eigenschaften und formalstrukturellen Merkmalen der Weiterbildungsorganisation in Verbindung bringen, wie es die bisherige Forschung zum Weiterbildungsmanagement gezeigt hat?

Zur Beantwortung dieser Fragestellungen wendet sich der Beitrag zunächst zentralen Grundannahmen der organisationspsychologischen Führungsforschung sowie der Governance- und Organisationsforschung zu, um systematisch Annahmen über Grunddimensionen der Führung, unterschiedliche Formen der Handlungskoordination sowie Eigenschaften und formalstrukturelle Merkmale von Organisationen zu entwickeln. ${ }^{2}$ In einem zweiten Schritt werden die aufgezeigten Forschungsfragen anhand einer fallvergleichenden Analyse mit den methodischen Mitteln der Grounded Theory nach Strauss und Corbin (1996) untersucht. Diese Variante der Grounded Theory hat sich für das eigene Untersuchungsinteresse nicht zuletzt deshalb empfohlen, da Strauss und Corbin ein auf unterschiedliche Erfahrungsgegenstände anwendbares, allgemeines Handlungsmodell in die Codierprozeduren der Grounded Theory integrieren, das Forschende darin unterstützt, handlungsbezogene Codes (hier das Führungsverständnis und die entsprechenden Handlungsorientierungen) systematisch mit Bedingungen (hier organisationale Rahmenbedingungen) und entstehenden Konsequenzen (hier durch die Führungsperson beförderte Koordinationsformen) in Beziehung zu setzen, ohne dabei den ergebnisoffenen Charakter des Verfahrens zu gefährden (vgl. Kelle und Kluge 2010, S. 63-64; Kelle 2007). So werden im Folgenden zwei maximal kontrastive Fälle mit Blick auf das jeweilige Führungsverständnis und die damit verbundenen Handlungsorientierungen, die seitens der Führungsperson favorisierten Koordinationsformen sowie mögliche bedingende Eigenschaften und formalstrukturelle Merkmale der Organisation analysiert. ${ }^{3}$ Der Beitrag schließt

\footnotetext{
2 Bei den skizzierten Annahmen der Führungs-, Governance- und Organisationsforschung handelt es sich um Ansätze, die größtenteils forschungsprozessbegleitend Eingang in die Untersuchung gefunden haben. Da anwendungsorientierte Beiträge zur Grounded Theory empfehlen, die für die Grounded Theory nach Strauss und Corbin charakteristische fortwährende Pendelbewegung zwischen Fachliteratur und Forschungsdaten zugunsten der Leserfreundlichkeit in Veröffentlichungen nicht mehr transparent zu machen, orientiert sich auch die Gliederung des vorliegenden Beitrags an den üblichen Konventionen für die Darstellung empirischer Forschungsbefunde (vgl. Goulding 2009, S. 389-390; Strauss und Corbin 1996, S. 38, 33).

3 Die Kennzeichnung als ,maximal“ kontrastiver Fallvergleich stützt sich auf das Modell der Reproduktionskontexte (vgl. Schrader 2011) als allgemeiner Heuristik. Das Modell differenziert verschiedene institutionelle Kontexte, die je spezifische Bedingungen der Sicherung des organisationalen Fortbestands hervorbringen. Die im Folgenden untersuchten Leitungspersonen sind in Weiterbildungsorganisationen beschäftigt, die sich insofern maximal voneinander unterscheiden, als die ÖFFENTLICH I einen öffentlichen Bildungsauftrag erfüllt und sich als öffentlich anerkannte Einrichtung zu einem erheblichen Teil über öffentliche Gelder finanziert (Kontext Staat), während die PRIVAT I privatwirtschaftliche Interessen verfolgt und sich ohne öffentliche Finanzierung unter Marktbedingungen reproduziert (Kontext Markt).
} 
mit einer fallvergleichenden Diskussion der Analyseergebnisse und fragt nach Ansatzpunkten für weitere Forschung.

Mit der vorliegenden kontrastiv-vergleichenden Fallstudie verbindet sich das theoriebildende Interesse, empirische Hinweise für den angenommenen Zusammenhang zwischen Führungsverständnis bzw. Orientierungen des Führungshandelns und organisationsinterner Handlungskoordination aus Sicht der Leitungspersonen zu identifizieren und mit Hilfe kontrastiver Fallvergleiche zu untersuchen, welche Variationen dieses Zusammenhangs empirisch auffindbar sind und wie sie mit organisationalen Rahmenbedingungen in Verbindung stehen.

\section{Theoretischer Hintergrund}

Etwa seit den 1940er Jahren beschäftigt sich die organisationspsychologische Führungsforschung systematisch mit dem Handeln von Führungskräften. Bis heute richtet sich dabei das Forschungsinteresse zahlreicher Beiträge auf die Dimensionalisierung des Führungsverhaltens, um empirisch bestätigte Grunddimensionen zu identifizieren, die für eine wirksame Einflussnahme auf andere Organisationsmitglieder bedeutsam sind (vgl. Weinert 2004; Wunderer 2009, S. 210). Für gewöhnlich wird zwischen drei Grunddimensionen des Führungsverhaltens unterschieden: das Einbeziehen der Mitarbeitenden in Entscheidungsprozesse (Entscheidungspartizipation), das Berücksichtigen von Bedürfnissen, Gefühlen und Interessen der Mitarbeitenden (Mitarbeiter- oder Beziehungsorientierung) sowie das Fokussieren auf die Zielerreichung und Aufgabenbewältigung (Aufgabenorientierung) (vgl. Weibler 2012, S. 344-345).

Für die Analyse der durch das Führungsverständnis und die individuellen Handlungsorientierungen beförderten Koordinationsformen haben sich vor allem solche Modelle als anschlussfähig erwiesen, die sich auf die erste Grunddimension - also den Grad der gewährten Entscheidungspartizipation - beziehen und dabei zwischen verschiedenen Führungsstilen unterscheiden (vgl. z. B. Lewin et al. 1939; Tannenbaum und Schmidt 1958; Vroom und Jago 1991; Neuberger 2002). Hierzu zählen beispielsweise:

- autoritäres Führen: Entscheidungs-, Weisungs- und Kontrollbefugnisse liegen einseitig allein beim/bei der Vorgesetzten;

- kooperatives Führen: Die Führungsperson teilt ihre Entscheidungskompetenz, indem sie ihre Mitarbeitenden in Entscheidungs- und Zielbildungsprozesse einbezieht; sie versucht (insbesondere in Konfliktsituationen) ausgleichend durch Ausund Verhandeln Einfluss zu nehmen und auf private und berufliche Interessen der Mitarbeitenden einzugehen;

- delegatives Führen: Die Führungskraft definiert (auch gemeinsam mit den Mitarbeitenden) relevante Ziele bzw. Aufgaben und überträgt die Aufgabenerledigung mit dafür erforderlichen Entscheidungsrechten an Mitarbeitende, wobei sie die Weisungs- und Kontrollmacht weiterhin für sich bewahrt; im Vergleich zur kooperativen Führung ist die Interaktionsdichte zwischen Führungskraft und Mitarbeitenden deutlich geringer (vgl. Hentze et al. 2005, S. 244-245, 253-257; Wunderer 2009, S. 228-229). 
Auch wenn die Ergebnisse der Führungsverhaltensforschung insbesondere mit Blick auf methodische Mängel (z. B. die geringe Validität der Messinstrumente; Mittelwertbildung über Items, Zeit und Mitarbeitende hinweg; vorwiegende Ermittlung des Führungsverhaltens anhand von subjektiven (Selbst-)Einschätzungen) durchaus kritisch diskutiert werden (vgl. Neuberger 2002), haben sich für die eigene Fallstudie vor allem Führungsstilunterscheidungen als eine hilfreiche Heuristik zur Weiterentwicklung der offen gebildeten Codes bewährt.

Rücken Prozesse der Handlungskoordination zwischen wechselseitig voneinander abhängigen sozialen Akteuren in den Mittelpunkt des Interesses, so hält die Governanceperspektive brauchbare (auf unterschiedliche Erfahrungsgegenstände anwendbare) Hilfsmittel für die Analyse von strukturellen Mechanismen und Formen des handelnden Zusammenwirkens bereit (vgl. Kussau und Brüsemeister 2007, S. 37, 40; Benz und Dose 2010b, S. 274). Während sich die Governanceforschung grundlegend für die Handlungskoordination zwischen individuellen und organisierten Akteuren interessiert, konzentriert sich der Ansatz der „Organisational Governance“ auf die organisationsinterne Handlungskoordination und zieht zu ihrer Analyse die begrifflichen Unterscheidungen der Governanceforschung heran (vgl. z. B. Schneider 2004, S. 178; Fombrum 1984).

Allerdings ist die Governanceperspektive auch nach mehr als zwei Jahrzehnten reger Forschungsaktivitäten in ihren Analysemitteln begrifflich noch relativ unscharf. So werden beispielsweise relativ dauerhafte, kollektiv geteilte Interaktionsstrukturen zur Handlungskoordination mal als basaler Mechanismus (vgl. Wiesenthal 2005, S. 231-232), mal als Governancetypen (vgl. Lütz 2010, S. 142), bisweilen als Modi (Blumenthal 2005, S. 1168) oder als institutionalisierte Formen der Governance (vgl. Brüsemeister 2007, S. 103-104; Benz und Dose 2010b, S. 256) bezeichnet. Uneinheitlich sind hierbei aber nicht nur die Bezeichnungen, sondern auch die damit verbundenen Inhalte. Während beispielsweise Benz und Dose aus einer politikwissenschaftlichen Perspektive zwischen Gemeinschaft, Wettbewerb, Hierarchie, Netzwerk und Verhandlung unterscheiden (vgl. Benz und Dose 2010b, 257-264), differenziert Williamson aus institutionenökonomischer Sicht zwischen Märkten, Firmen, Hybridformen und Bürokratie (vgl. Williamson 1985).

Trotz dieser nach wie vor bestehenden Uneinheitlichkeit zeichnen sich aber dennoch folgende Annahmen als konsensfähig ab: Soziale Akteure sind in der Umsetzung ihrer jeweiligen Handlungsziele niemals völlig unabhängig, sondern fortwährend auf die Handlungsabstimmung mit anderen Akteuren angewiesen. Dabei kann ein gemeinsames Handeln durch ,Nachahmung auf der Grundlage von Beobachtung“, durch „Anpassung auf der Grundlage von Beeinflussung“ oder durch „Vereinbarungen auf der Grundlage von Verhandlungen“ zustande kommen. Sozial eingebettet ist dieses gemeinsame Handeln in institutionalisierte Interaktionsstrukturen, die sich in je empirisch zu bestimmenden Kombinationen realisieren können und für gewöhnlich auf den basalen Koordinationsformen Hierarchie, Markt (bzw. Wettbewerb), Gemeinschaft und Netzwerk beruhen (Benz und Dose 2010b, S. 251-264; vgl. auch Blumenthal 2005, S. 1169; Kussau und Brüsemeister 2007, S. 37-41). Die in Organisationen vorherrschende Interaktionsstruktur der Hierarchie basiert auf dem Prinzip der Über- und Unterordnung und auf formalen Regeln. Markt bzw. Wettbewerb erfordert „ein geregeltes Verhältnis der Konkurrenz“ und wechselsei- 
tiges Beobachten mit Blick auf Geleistetes. Gemeinschaften beruhen auf sozialer Gruppenkohäsion und geteilten Grundhaltungen. Netzwerke zeichnen sich gerade durch die Abwesenheit streng „formal geregelte[r] Interaktionsbeziehungen“ aus; gemeinsames Handeln wird hier durch wechselseitiges Vertrauen hervorgebracht (Benz und Dose 2010b, S. 257-264, 254; vgl. auch Kussau und Brüsemeister 2007, S. 40-41).

Insbesondere diese zuletzt skizzierte Systematisierung basaler Koordinationsformen nach je spezifischen Leitprinzipien hat im Rahmen der eigenen Fallstudie als orientierende Aufmerksamkeitsrichtung für das Auffinden von Koordinationsformen im empirischen Material Berücksichtigung gefunden.

Mit Blick auf die organisationalen Rahmenbedingungen hat schließlich die sozialwissenschaftliche Organisationsforschung hilfreiche Unterscheidungen entwickelt. Im Anschluss an situative Ansätze der Organisationsforschung lassen sich für die Innenwelt der Organisation organisationale Eigenschaften von formalstrukturellen Organisationsmerkmalen abgrenzen. Während zu den Eigenschaften der Organisation etwa ihre Größe (oftmals operationalisiert als Zahl der Mitarbeitenden), ihr Alter oder ihre Rechtsform zählen, beschreiben beispielsweise Arbeitsteilung, dadurch notwendig werdende Instrumente der Koordination sowie die Aufbau- und Ablauforganisation (z. T. auch als Konfiguration bezeichnet) formalstrukturelle Merkmale im engeren Sinne (vgl. Kieser und Walgenbach 2010; Bea und Göbel 2010).

Im Folgenden interessiert nun weder die spezifische Analyseperspektive der situativen Ansätze, die formalstrukturelle Unterschiede auf situative Unterschiede zurückzuführen versuchen, noch sollen die Koordinationsinstrumente der sozialwissenschaftlichen Organisationsforschung zugrunde gelegt werden. Vielmehr richtet sich das Hauptaugenmerk auf durch das Führungsverständnis bzw. durch entsprechende Handlungsorientierungen beförderte hierarchische und nicht-hierarchische Formen der organisationsinternen Handlungskoordination. Da die in der sozialwissenschaftlichen Organisationsforschung diskutierten Koordinationsinstrumente nicht-hierarchische Koordinationsformen kaum differenzieren (vgl. Kieser und Walgenbach 2010, S. 100-127), finden hier die zuvor skizzierten Unterscheidungen der Governanceforschung an ihrer Stelle als übergeordnete Heuristik Berücksichtigung. Gleichwohl werden auch im Rahmen des vorliegenden Beitrags Eigenschaften und formalstrukturelle Merkmale der Weiterbildungsorganisationen betrachtet. Denn in Orientierung an der bisherigen Weiterbildungsmanagementforschung wird angenommen, dass die identifizierten Ausprägungen des Führungsverständnisses und der damit korrespondierenden Handlungsorientierung mit den jeweiligen organisationalen Rahmenbedingungen in Verbindung stehen. Hierfür wird die Differenzierung der situativen Ansätze zwischen Eigenschaften und formalstrukturellen Merkmalen der Organisation herangezogen, um eben diese organisationalen Bedingungen systematisch innerhalb des Datenmaterials aufsuchen zu können.

\section{Methodisches Vorgehen}

Die im Folgenden dargestellten Befunde einer vergleichenden Fallstudie (vgl. Hering und Schmidt 2014; Yin 2009) beruhen auf einer mikroanalytischen Auswertung von 
Leitfadeninterviews mit Leitungspersonen in der Erwachsenen- und Weiterbildung sowie von Organisationsdaten (z. B. Homepage, Satzung, Organigramm der jeweiligen Einrichtung) in Orientierung an den Grundprinzipien der Grounded Theory nach Strauss und Corbin (1994, 1996).

\subsection{Methodenauswahl}

Für die Methodenwahl waren vor allem folgende Überlegungen ausschlaggebend: Zum einen sollten die Einzelinterviews mit Leitungspersonen durch öffentlich zugängliche Organisationsdaten und Dokumente ergänzt werden, um die Reichhaltigkeit des empirischen Materials zu erhöhen und ein methodisch kontrolliertes Fremdverstehen zu erleichtern. Anders als manch andere Methode der qualitativen Sozialforschung erlaubt die Grounded Theory ausdrücklich eine solche Kombination unterschiedlicher Materialtypen, sofern für die herangezogenen Daten begründet angenommen werden kann, dass sie einen Beitrag zur (Weiter-)Entwicklung der ermittelten Kategorien leisten (vgl. Glaser und Strauss 2008, S. 72-76; Strauss 1998, S. 25). Zum anderen lenken die pragmatistisch-interaktionistischen Traditionslinien der Grounded Theory insbesondere in der durch Strauss und Corbin vertretenen Variante den Blick auf alltägliche Handlungszusammenhänge, indem in fortgeschrittenen Phasen des Analyseprozesses mit Hilfe des sogenannten Codierparadigmas interessierende Handlungsanforderungen (oder allgemeiner soziale Phänomene) mit ihren Bedingungen, gewählten Strategien und entstehenden Konsequenzen in Beziehung gesetzt werden (vgl. Strauss 1998, S. 57; Strauss und Corbin 1996, S. 9, 75-85). Mit diesem basalen Handlungsmodell leiten Strauss und Corbin Forschende dazu an, allgemeine Annahmen über ein Phänomen und damit verbundene Bedingungen, Strategien und Konsequenzen zu bilden sowie Variationen dieses Zusammenhangs innerhalb des Forschungsfeldes gezielt aufzusuchen. Vor diesem Hintergrund erweist sich die Grounded Theory nach Strauss und Corbin auch deshalb als angemessenes methodisches Verfahren, weil sie mit ihren methodischen Mitteln den hier interessierenden Zusammenhang zwischen Führungsverständnis bzw. Handlungsorientierungen und organisationsinterner Handlungskoordination auch mit Blick auf organisationale Rahmenbedingungen systematisch zu analysieren erlaubt.

$\mathrm{Zu}$ begründen ist allerdings, warum Interviews als maßgebliche Datengrundlage herangezogen werden, wenn sich doch das Forschungsinteresse auf die durch das Führungsverständnis und die entsprechenden Handlungsorientierungen beförderte Handlungskoordination richtet und auch die Grounded Theory nach Strauss und Corbin ein basales Handlungsmodell in ihren Auswertungsprozess integriert.

Die Entscheidung für Interviews als maßgeblicher Datengrundlage liegt methodologisch vor allem im Wirklichkeitsverständnis der qualitativen Sozialforschung im Allgemeinen und der Grounded Theory im Besonderen begründet. Im Anschluss an interaktionistisch-pragmatistische Überlegungen ist die soziale Wirklichkeit eine interpretierte Wirklichkeit, d. h. individuelles Handeln, aber auch das handelnde Zusammenwirken mehrerer Akteure in einer Organisation oder gesellschaftliche Ordnungen beruhen maßgeblich auf interaktiven Gestaltungs- und Deutungsleistungen der beteiligten Akteure (vgl. Blumer 2004). Dabei ist im Sinne des US-amerikanischen Pragmatismus von einem Zusammenspiel der Sicht- und Handlungswei- 
sen auszugehen. Welche Bedeutung Ereignisse der sozialen Wirklichkeit haben, ist einerseits auf die jeweils gemachten Handlungserfahrungen zurückzuführen und andererseits wirken diese erfahrungsgebundenen Bedeutungszuschreibungen selbst wiederum auf das Handeln und damit verbundene Wahlentscheidungen zurück (vgl. Peirce 1991). Vor diesem Hintergrund lässt sich nun annehmen, dass auch die interviewten Leitungspersonen in ihren Erzählungen nicht nur Vorstellungen davon offenbaren, wie Führung als absichtsvolle Einflussnahme auf andere zugunsten des gemeinsamen Handelns möglich ist, sondern immer auch zentrale Orientierungen ihres Handelns preisgeben (vgl. Geulen 1982). Sie stellen insofern wichtige Schlüsselpersonen für die Analyse der durch Führung beförderten organisationsinternen Koordinationsformen dar, als ihre Interpretationsleistungen qua zugewiesener Positionsmacht über größere Durchsetzungschancen verfügen (vgl. Kieser und Walgenbach 2010, S. 167).

\subsection{Sampling}

Im Rahmen der Fallstudie wurden nach einer Erprobung des Leitfadens zehn Leitfadeninterviews mit Leitungspersonen in Weiterbildungsorganisationen geführt, die unter unterschiedlichen Kontextbedingungen agieren. Um eine grundsätzliche Vergleichbarkeit der Fälle gewährleisten zu können, sind nur solche Leitungspersonen um ein Interview gebeten worden, die formal neben der Personalführung auch mit der Aufgabe der Organisationsführung betraut sind, da sie im Vergleich zu Projekt- oder Abteilungsleitungen über privilegiertere Möglichkeiten der Einflussnahme auf die organisationsinterne Handlungskoordination verfügen (vgl. Weibler 2012, S. 149). Darüber hinaus wurden ausschließlich Weiterbildungsorganisationen einbezogen, die selbst ein Weiterbildungsangebot vorhalten, so dass Dachorganisationen oder Zentralen mit reinen Verwaltungsaufgaben unberücksichtigt geblieben sind. Die Unterschiedlichkeit der (organisationalen) Rahmenbedingungen wurde empirisch vor allem über das organisationale Merkmal Trägerschaft erschlossen, da der Träger zum einen für die formalrechtlichen und finanziellen Grundlagen Sorge trägt, zum anderen aber auch den Organisationszweck und die inhaltlichen (Bildungs-)Ziele beeinflusst (vgl. Gnahs 2010, S. 288), weshalb ihm in der Weiterbildungsforschung strukturelle Ordnungsmächtigkeit zugeschrieben wird (vgl. Schlutz 1997). Ergänzend sind zudem der Erwerbszweck (gemeinnützig vs. erwerbswirtschaftlich), die Zahl der Mitarbeitenden und die Anerkennung nach geltendem Weiterbildungsgesetz herangezogen worden. Insgesamt setzt sich der Interviewpool aus fünf öffentlich geförderten Einrichtungen, drei Weiterbildungsorganisationen, die in der Trägerschaft gesellschaftlicher Interessengruppen stehen, und zwei privatwirtschaftlichen Weiterbildungsanbietern zusammen.

Für die konkrete Fallauswahl sieht die Grounded Theory im Idealfall ein verlaufsoffenes, iteratives Vorgehen vor (theoretical sampling), indem durch eine unmittelbar an die Erhebung des ersten Falls anschließende Analysephase eng mit dem empirischen Material verbundene Codes entwickelt werden, die anschließend als Kriterien für die nächste Auswahlentscheidung dienen (vgl. Strauss und Corbin 1996, S. 148-159). Forschungspraktisch stellt das theoretical sampling Forschende allerdings vor die Herausforderung, wie diese verlaufsoffene, schrittweise Fallaus- 
Tab. 1 Exemplarische Codes für die Vorbereitung von Samplingentscheidungen

\begin{tabular}{|c|c|c|}
\hline Codes & Codeeigenschaften & Kategorie \\
\hline $\begin{array}{l}\text { Führung als inhalts-, aber auch be- } \\
\text { ziehungsorientierte Kommunikation }\end{array}$ & nicht-vorhanden - vorhanden ${ }^{\mathrm{a}}$ & $\begin{array}{l}\text { Merkmale des } \\
\text { Führungsverständnisses }\end{array}$ \\
\hline $\begin{array}{l}\text { Regeln als selbstverständlicher } \\
\text { Bestandteil von Führung }\end{array}$ & nicht-vorhanden - vorhanden & \\
\hline$\cdots$ & $\cdots$ & \\
\hline $\begin{array}{l}\text { gemeinsamer Teambeschluss (bei } \\
\text { grundsätzlich neuen Aufgaben) }\end{array}$ & nicht-vorhanden - vorhanden & $\begin{array}{l}\text { Merkmale kooperativer } \\
\text { Handlungsorientierung }\end{array}$ \\
\hline $\begin{array}{l}\text { Regeln festlegen durch gemeinsa- } \\
\text { mes Vereinbaren }\end{array}$ & nicht-vorhanden - vorhanden & \\
\hline $\begin{array}{l}\text { Teamgespräche vermittelnd/ } \\
\text { ausgleichend führen }\end{array}$ & nicht-vorhanden - vorhanden & \\
\hline $\begin{array}{l}\text { gemeinsame Werteorientierungen/ } \\
\text { Grundhaltungen }\end{array}$ & nicht-vorhanden - vorhanden & $\begin{array}{l}\text { Merkmale der } \\
\text { Koordinationsform }\end{array}$ \\
\hline $\begin{array}{l}\text { Grenzziehung nach außen (z. B. } \\
\text { gegenüber der Buchhaltung) }\end{array}$ & nicht-vorhanden - vorhanden & Gemeinschaft \\
\hline$\ldots$ & $\ldots$ & \\
\hline $\begin{array}{l}\text { Zahl der Mitarbeitenden } \\
\text {... }\end{array}$ & $\begin{array}{l}\text { niedrig - hoch } \\
\text {... }\end{array}$ & $\begin{array}{l}\text { Eigenschaften der } \\
\text { Organisation }\end{array}$ \\
\hline Stellenbeschreibungen & $\begin{array}{l}\text { nicht-vorhanden - vorhanden } \\
\text {... }\end{array}$ & $\begin{array}{l}\text { Formalstrukturelle } \\
\text { Merkmale der Organisation } \\
\text { (Aufbauorganisation) }\end{array}$ \\
\hline $\begin{array}{l}\text { Gesprächsroutinen innerhalb der } \\
\text { Arbeitsgruppe } \\
\text {... }\end{array}$ & $\begin{array}{l}\text { nicht-vorhanden - vorhanden } \\
\text { zeitlicher Abstand: } \\
\text { gering - hoch } \\
\text { Teilnahmeverbindlichkeit: } \\
\text { gering - hoch } \\
\text {... }\end{array}$ & $\begin{array}{l}\text { Formalstrukturelle Merkma- } \\
\text { le der Organisation (Ablauf- } \\
\text { organisation) }\end{array}$ \\
\hline$\ldots$ & $\ldots$ & $\ldots$ \\
\hline
\end{tabular}

${ }^{\mathrm{a}}$ Kursiv hervorgehobene Codeeigenschaften treffen auf den analysierten Fall zu

wahl angesichts der zeitlichen Begrenzung von Feldzugängen und Forschungsprojekten angemessen umgesetzt werden kann (vgl. Truschkat et al. 2011, S. 370-371; Locke 2001, S. 111). Hinzu kommt, dass die als Samplingkriterien herangezogenen Codes in der Regel ,,per Augenschein“ unzugänglich sind, so dass vor der Erhebung oftmals keine verlässliche Aussage darüber getroffen werden kann, ob eine Person tatsächlich Merkmalsträger im Sinne des nächsten gewünschten Falls ist (vgl. Strübing 2013). Um diesen Schwierigkeiten zu entgehen, beruht die eigene Fallstudie auf einer forschungspragmatischen Variante des theoretical samplings, die sowohl in der anwendungsbezogenen Sekundärliteratur (vgl. z. B. Strübing 2013, Truschkat et al. 2011) als auch von Strauss und Corbin (vgl. 1996, S. 164) selbst aufgezeigt wird: Dabei werden zu Beginn des Forschungsprozesses eine Reihe von Interviews geführt, innerhalb dieses generierten Interviewpools die Prinzipien des theoretical samplings umgesetzt und auf dieser Grundlage schließlich begründete Entscheidungen für die Weiterentwicklung des Samples getroffen.

Während für die Auswahl des ersten Falls noch methodische Kriterien wie die „Reichweite“ und „Spezifität“ des Materials (vgl. Hopf 1978, S. 107) ausschlagge- 
bend sind, orientieren sich die weiteren „Fallziehungen“ an den sukzessive entstehenden Codes. Tab. 1 zeigt beispielhaft ausgewählte Codes eines Falls und ihre Bündelung zu abstrakteren Kategorien, die ein systematisches Aufsuchen von zunächst strukturell unterschiedlichen und in späteren Analysephasen strukturell ähnlichen Fällen ermöglichen (vgl. Strauss und Corbin 1996, S. 150).

\subsection{Qualitätskriterien}

Abgesehen von einer für die qualitative Sozialforschung charakteristischen reflexiven Haltung gegenüber dem eigenen Forschungs- und Erkenntnisprozess, die forschungspraktisch beispielsweise in der kritischen Analyse der Interviewführung, des Verhältnisses von argumentierenden und erzählenden Interviewpassagen oder der Umsetzung formalisierter Analyseschritte Berücksichtigung gefunden hat, wurden zur Qualitätssicherung das Fallstudiendesign, der Leitfaden, Samplingentscheidungen und die mit Hilfe von MAXQDA aufbereiteten Codierergebnisse in Forschungskolloquien, im Rahmen von Methodenworkshops und in Beratungsgesprächen diskutiert.

\section{Ergebnisse}

Mit Blick auf die zu Beginn skizzierten Forschungsfragen werden im Folgenden Ergebnisse eines maximal kontrastiven Fallvergleichs zwischen dem Leiter A05 der Weiterbildungsorganisation ÖFFENTLICH I und dem Leiter A10 der Weiterbildungsorganisation PRIVAT I dargelegt. Beide Interviews wurden in den Büroräumen der Leitungspersonen mit einer Interviewzeit von jeweils etwa 80 Minuten geführt, vollständig transkribiert und mikroanalytisch ,line-by-line“ ausgewertet. Für den Fall des Leiters A05 sind 157 Codes und für den Fall des Leiters A10 287 Codes entwickelt und anschließend zu übergeordneten Konzepten bzw. Kategorien verdichtet worden.

Leiter A05 der Weiterbildungsorganisation ÖFFENTLICH I. Bei der ÖFFENTLICH I handelt es sich um eine öffentlich geförderte, gemeinnützige Einrichtung, die als Amt in eine kommunale Trägerstruktur eingebunden ist. Mit acht hauptberuflichen Mitarbeiterinnen und etwa 180 neben- oder freiberuflichen Kursleitenden verfügt sie über eine für den Weiterbildungsbereich charakteristische, disparate Mitarbeiterstruktur, die sich aus einer vergleichsweise geringen Zahl an (mehrheitlich weiblichen) Festangestellten und einem deutlich höheren Anteil an Honorarkräften zusammensetzt (vgl. Martin und Langemeyer 2014, S. 46, 48). Ablauforganisatorisch sind in der ÖFFENTLICH I gemessen an der Zahl festangestellter Mitarbeiterinnen relativ ausgeprägte Gesprächsroutinen etabliert. Zum einen findet vierzehntäglich eine Besprechung zwischen dem Leiter und den drei hauptamtlichen pädagogischen Mitarbeiterinnen statt, in der über pädagogische und grundsätzliche Fragen beraten wird. Zum anderen ist wöchentlich versetzt eine Gesamtteamsitzung für das festangestellte Personal ebenfalls vierzehntäglich anberaumt, um allen die Möglichkeit zu geben, sich zu informieren, Fragen einzu- 
bringen und sich am organisationalen Geschehen zu beteiligen. Für den Austausch über die individuelle Arbeitssituation und -zufriedenheit werden jährlich Mitarbeitergespräche geführt und ein- bis zweimal im Jahr wird überdies ein Klausurtag eingelegt, um sich innerhalb der Arbeitsgruppe strategisch über Zielperspektiven, Entwicklungsmöglichkeiten und grundsätzliche Probleme zu verständigen.

In den Erzählungen über seinen beruflichen Alltag hebt der Leiter A05 mehrmals während des Interviews auf Regeln als eine notwendige Voraussetzung für ein gemeinsames Handeln innerhalb der Arbeitsgruppe ab. Dabei verweist er zum einen auf formalisierte Regeln wie den Geschäftsverteilungsplan oder Stellenbeschreibungen, die durch schriftlich fixierte Zuständigkeits- und Weisungsbefugnisse eine hierarchische Ordnung im Inneren der Organisation herstellen. Zum anderen nimmt er auf formale Regeln für Führung und Kommunikation Bezug, die ohne Schriftlichkeit auskommen, innerhalb der Arbeitsgruppe aber dennoch über eine ähnlich hohe Verbindlichkeit verfügen (vgl. Kieser und Walgenbach 2010, S. 157). In diesem Zusammenhang scheint der Mechanismus der Handlungskoordination für ihn eine besondere Bedeutung zu haben; wiederholt stellt er heraus, dass Regeln innerhalb der Arbeitsgruppe ,,vereinbart“ werden.

\begin{abstract}
„Also ich glaube, dass wir ganz gut als Team arbeiten. Das setzt aber auch voraus, dass wir klare Stellenbeschreibungen und Regeln haben. Wir haben einen Geschäftsverteilungsplan, wir haben (.) Stellenbeschreibungen und wir haben im Zusammenhang mit einem schon viele Jahre währenden Qualitätsprozess [.] haben wir auch klare Regeln für Führung und Kommunikation vereinbart. Vereinbart. (I lacht) Und wir halten uns auch an diese Regelung [Das Telefon klingelt.] und können dann auch gut im Team miteinander arbeiten“ (A05, Z. 255-266). ${ }^{4}$
\end{abstract}

Vereinbarungen können als das Ergebnis wechselseitigen Verhandelns verstanden werden, die Verhandlungswilligkeit und -fähigkeit der Beteiligten voraussetzen (vgl. Schimank 2010, S. 305). Beides scheint auf Seiten des Leiters A05 grundsätzlich gegeben zu sein. So erzählt er in mehreren Interviewpassagen davon, dass innerhalb der Arbeitsgruppe sowohl über die (Kommunikations-)Regeln der täglichen Zusammenarbeit als auch über Führung selbst verhandelt wird. Dass mit diesen mündlich vereinbarten Regeln nicht nur die Handlungsspielräume seiner Mitarbeiterinnen, sondern zugleich auch seine eigenen einschränkt werden können (vgl. Crozier und Friedberg 1979, S. 75), scheint er nicht als grundlegende Bedrohung seiner Einflusspotenziale wahrzunehmen. Die durchgängige Verwendung der ersten Person Plural deutet eher darauf hin, dass er sich in der täglichen Zusammenarbeit als reguläres „Team“-Mitglied betrachtet, das an vereinbarte Regeln gebunden ist und dessen Handeln ebenso zum Thema gemacht werden kann wie das aller anderen. An späterer Stelle wird zudem deutlich, dass A05 auch eine mit seinen Mitarbeiterinnen geteilte Entscheidungsmacht nicht als Beeinträchtigung der strukturell verankerten

\footnotetext{
4 Das Transkriptionssystem orientiert sich größtenteils an den entsprechenden Empfehlungen von Kuckartz et al. (2008). In den Interviewausschnitten vorkommende Transkriptionszeichen: (.) kurzes Aussetzen, kurze Pause; Vereinbart Betonung; (I lacht) (Laut-)Äußerungen der jeweils anderen Person, [Das Telefon klingelt.] situative Zusatzinformationen.
} 
Hierarchie innerhalb der Arbeitsgruppe wahrnimmt. Vielmehr hebt er hervor, dass auch Leitungskräfte situativ abwägen können, ob eine Entscheidung von ihnen allein oder per Mehrheitsentscheid innerhalb der Arbeitsgruppe getroffen wird.
„Also ich denke, dass man in einer leitenden Funktion schon auch eine beson- dere Verantwortung hat und zu dem stehen sollte, was man selbst denkt und mag, auch wenn andere Meinung auftaucht. Man kann dann auch an irgend- einer Stelle sagen: 'Gut ich bin hier in der Minderheit, das ist jetzt nicht so existentiell'“ (A05, Z. 362-368).

$\mathrm{Zu}$ einer gemeinsamen Entscheidungsfindung kann es aus Sicht des Leiters A05 allerdings nicht nur kommen, wenn er den Entscheidungsgegenstand als ,nicht so existentiell“" einschätzt; auch grundlegende Veränderungen des Leistungsprogramms der ÖFFENTLICH I erfordern für ihn einen gemeinsamen Beschluss durch die Arbeitsgruppe. Dabei scheint das Prinzip einer situativ geteilten Einflussnahme Ausdruck einer gemeinsam vertretenen Grundhaltung innerhalb der Arbeitsgruppe zu sein; jedenfalls ist A05 davon überzeugt, dass eine Missachtung dieses Prinzips von seinen Mitarbeiterinnen und ihm selbst in gleicher Weise bewertet wird, sie ,würde [...] nicht akzeptiert".

\begin{abstract}
„Wir haben auch hier festgelegt, dass wir vor der Übernahme neue Aufgaben in der ÖFFENTLICH I - das ist jetzt nicht ein einzelner Englischkurs oder so was, sondern grundsätzlich neue Aufgaben - im Team besprechen und gemeinsamen Beschluss dazu fassen. Also eine isolierte Entscheidung, dass ich sage: 'So, ich habe jetzt also neue Aufgaben. Das und das fällt jetzt weg und das und das machen wir künftig neu', das würde hier nicht ins System passen und würde auch nicht akzeptiert und das finde ich auch gut und richtig so, denn es käme nichts Gutes dabei raus, wenn ich als Leiter diktatorisch etwas verfügen würde“" (A05, Z. 375-387).
\end{abstract}

Eine relativ reibungslose Handlungsabstimmung innerhalb der Arbeitsgruppe zu ermöglichen, scheint für den Leiter A05 keine besondere Herausforderung darzustellen; zumindest thematisiert er arbeitsgruppeninterne Konflikte nur an einer Stelle als „Spannung, die natürlicherweise auch entsteht in einem Lebens- und Geschäftsablauf" (A05, Z. 495-497). Anschließend erzählt er von seinem Umgang mit widerstreitenden Interessen der Mitarbeiterinnen hinsichtlich der internen Arbeitsteilung. Bedingt durch einen veränderten Stellenwert von Arbeit innerhalb der individuellen Berufsbiografie und/oder bedingt durch ein angesichts veränderter Umwelterwartungen sich wandelndes Aufgabenspektrum der ÖFFENTLICH I kann eine Umverteilung der bisherigen Arbeitsorganisation notwendig werden, die innerhalb der Arbeitsgruppe bisweilen zu Unstimmigkeiten geführt hat. Dass die Bedeutung von Arbeit für die individuelle Lebensführung je nach Lebenslage mal geringer, mal höher ausfallen kann, beobachtet A05 mit großem Verständnis.

„Und so gibt es bei jedem Mensch und bei jeder Person Phasen, wo man sich voll in die Arbeit freiwillig schmeißt, wo man unfreiwillig unter Umständen das macht, wo man auch einmal sagt: 'Jetzt will ich, muss ich reduzieren. So geht es nicht weiter'“ (A05, Z. 523-529). 
Er achtet darauf, individuelle und organisationale Interessen in Einklang zu bringen und schildert Gespräche mit den Beteiligten, in denen er versucht, zu vermitteln und Interessenkonflikte durch wechselseitiges Verhandeln auszugleichen (,Also diese Spannungen die dadurch auch in einem Team entstehen, die müssen bewusst zum Ausgleich gebracht werden“, A05, Z. 538-541).

Aus führungspsychologischer Sicht stellen eben diese Achtsamkeit für Bedürfnisse und Anliegen der Mitarbeitenden, das Bestreben, auf Konflikte ausgleichend und vermittelnd einzuwirken und die zuvor beschriebene Akzeptanz einer (situativ) geteilten Einflussnahme charakteristische Merkmale für kooperatives Führen dar (vgl. Wunderer 2009, S. 219, 228; Hentze et al. 2005, S. 254-255).

Leiter A10 der Weiterbildungsorganisation PRIVAT I. Die PRIVAT I ist eine $\mathrm{GmbH}$ mit eigenwirtschaftlichem Erwerbszweck, die keine öffentliche Förderung erhält, sondern ihre Dienstleistungen und Produkte ausschließlich unter Marktbedingungen vertreibt. Zu ihrem Leistungsangebot zählen offen und als Inhouse-Seminare durchgeführte Trainings, Organisationsberatungsmaßnahmen und Softwaretools. Die PRIVAT I verfügt über vier Geschäftsführer, unter denen der Leiter A10 als Hauptanteilseigner der Gesellschaft jedoch mit der größten Entscheidungskompetenz ausgestattet ist. Insgesamt sind in der PRIVAT I zum Zeitpunkt des Interviews etwa 100 festangestellte Mitarbeiterinnen und Mitarbeiter beschäftigt, wobei dem Leiter A10 die direkte Personalverantwortung für 25 Mitarbeitende obliegt. Die Ablauforganisation zeichnet sich durch eindeutig rhythmisierte Gesprächsroutinen aus, die in größeren zeitlichen Abständen stattfinden. Die Geschäftsführer treffen sich monatlich zur Geschäftsführersitzung. Vierteljährlich sind jeweils separate Sitzungstermine mit den Produktmanagern und Produktmanagerinnen, den Key-AccountManagern und -Managerinnen und den Trainern und Trainerinnen angesetzt, in denen z. B. über die Zielgruppenansprache oder das Produktangebot beraten wird, aber auch zusätzliche Aufgaben durch A10 verteilt werden. Zudem werden einmal im Jahr Zielvereinbarungsgespräche geführt, die dem beidseitigen Feedback, der Vereinbarung von im nächsten Jahr zu erreichenden Zielen und der Verhandlung über die Vergütung dienen.

Ähnlich wie der Leiter A05 thematisiert auch der Leiter A10 die Interaktionsbeziehungen mit seinen Mitarbeitenden im Wesentlichen mit Blick auf ihre Beteiligung. Anders als A05, der maßgeblich auf die Entscheidungspartizipation der Arbeitsgruppe rekurriert, bezieht sich A10 allerdings vorwiegend auf individuelle Beteiligungsmöglichkeiten, indem Aufgaben einzeln mit den jeweiligen Mitarbeitenden verhandelt und die erforderlichen Befugnisse für die Aufgabenerledigung anschließend vertikal übertragen werden. Ein ,entscheidendes“ Führungsinstrument stellen für ihn jährliche Zielvereinbarungsgespräche dar, die auf ein an Delegation orientiertes Führungsverständnis und eine entsprechende Handlungsorientierung hinweisen (vgl. Kieser und Walgenbach 2010, S. 156; Wunderer 2009, 230-231).

„Und die entscheidenden Sachen sind eigentlich Zielvereinbarungsgespräche. Also die sind eben, jetzt stehen die an, im Juni habe ich die alle vereinbart. Jetzt wurden die für zwei Stunden anberaumt, aber meistens dauern die drei/ 
vier Stunden. Weil es ist halt rückwirkend einmal gesehen, wie ist das Jahr gelaufen und was nehmen wir uns für das nächste Jahr vor" (A10, Z. 448-456).

Mit den Zielvereinbarungsgesprächen rückt A10 je individuelle Tauschbeziehungen zwischen der betreffenden Mitarbeiterin bzw. dem betreffenden Mitarbeiter und ihm in den Fokus, da jeweils einzeln über Ziele, Zielerreichung und einzutauschende Gegenleistungen verhandelt wird. Obgleich dieses Tauschinteresse wechselseitig bestehen und gemeinsam über Leistung und Gegenwert verhandelt werden mag, nivellieren Zielvereinbarungsgespräche dennoch nicht die hierarchisch geprägte Interaktionsstruktur innerhalb der Arbeitsgruppe - eher im Gegenteil: Da sie mit einer Überprüfung der Aufgabenerledigung einhergehen und der Vorgesetzte je nach Zielerreichungsgrad über den Umfang der gewährten Gegenleistung entscheiden kann, tragen Zielvereinbarungen für gewöhnlich zu Stabilisierung einer hierarchisch organisierten Handlungskoordination bei (vgl. Nerdinger 2012, S. 202-203).

Eine solche Aktualisierung der Hierarchie in der face-to-face-Interaktion kann möglicherweise gerade in der PRIVAT I unterstützend wirken, weil die für eine hierarchische Handlungsabstimmung notwendige Kommunikation unter Anwesenden hier nicht regelmäßig gewährleistet ist (vgl. Benz und Dose 2010b, S. 261). Ablauforganisatorisch wird ein Großteil des Leistungsangebots in der PRIVAT I als Inhouse-Seminar für Unternehmen erbracht, so dass der Leiter A10 im Vergleich zu A05 deutlich seltener die Gelegenheit zu haben scheint, seine Führungsbeziehungen in direkter Interaktion mit seinen Mitarbeitenden zu gestalten. Diese vergleichsweise geringere Interaktionsdichte innerhalb der Arbeitsgruppe lässt sich in Orientierung an Befunden der psychologischen Führungsforschung als ein weiteres Indiz für einen delegativen Führungszusammenhang interpretieren (vgl. Wunderer 2009, S. 228).

„Und wir haben - das habe ich selbst auch extrem gut kennen gelernt bei uns durch den Gründer und habe das einfach fortgeführt, eine Zielvereinbarungskultur, kann man wirklich sagen. Also da müssen Sie sich vorstellen, die Trainer, die machen teilweise 120/140 Tage, fakturierbare Tage beim Kunden, die sind also nie da. Wir haben da vorne das Großraumbüro, aber die sind halt von Montag bis Freitag entweder bei einer Großbank oder einem Touristikkonzern oder was weiß ich, wo die sind. Und deshalb sehe ich die jetzt in der Führung eigentlich alle Nase lang einmal. Und deshalb ist das natürlich jetzt von der Führung her eine sehr, sehr, sehr lange Leine“ (A10, Z. 435-448).

Im Vergleich zu A05 schildert der Leiter A10 die Koordination der Arbeitsgruppe als weniger reibungslos. So haben beispielsweise einzelne Mitarbeitende im Zuge der vierteljährlich stattfindenden Teamsitzungen ihren Unmut darüber zum Ausdruck gebracht, dass zusätzliche Aufgaben von einigen Arbeitsgruppenmitgliedern zugunsten ihrer formalen Zielvorgaben vernachlässigt werden. Und auch er selbst beobachtet Nachlässigkeiten und die Priorisierung persönlicher Ziele bei einzelnen.

„Also, wie gesagt, ich denke da zur Zeit viel darüber nach, weil mir jetzt so aufgefallen ist in den letzten Meetings, dass da viele, viele Punkte einfach liegengeblieben sind. Und auch wenn ich mit zwei/drei Kollegen gesprochen habe, die sagten: 'Also das ist schon ärgerlich also jetzt hier. Ich habe jetzt 
hier mich gemeldet und habe meine Sache gemacht, andere nicht'. Und das ist ja so eine Art, ja im Prinzip dann auch Kollegialität, also, dass jeder seinen

Teil dazu beiträgt“ (A10, Z. 1695-1704).

Obgleich Ergebnisse der Führungsforschung zeigen, dass sich Zielvereinbarungen positiv auf die Leistungsbereitschaft und Arbeitszufriedenheit auswirken können (vgl. Rodler und Kirchler 2002, S. 65-66), thematisiert A10 für das vorwiegend delegative Führen durch Zielvereinbarungsgespräche gepaart mit der geringen Interaktionsdichte in der PRIVAT I indirekt auch negative Folgen. Die gemeinsamen Teamsitzungen scheinen von den Mitarbeitenden jedenfalls auch dazu genutzt zu werden, sich wechselseitig als Konkurrenten um einsetzbare Ressourcen zur Verfolgung eigener Ziele zu beobachten.

\section{Diskussion}

Mit dem Führungskonzept richtete sich das Hauptaugenmerk des vorliegenden Beitrags auf die direkte Handlungsabstimmung zwischen Vorgesetzten und Mitarbeitenden aus Sicht der jeweiligen Leitungsperson. Dabei interessierte vor allem, wie Leitungspersonen durch ihr Führungsverständnis bzw. ihre entsprechenden Handlungsorientierungen hierarchische und nicht-hierarchische Formen der Handlungskoordination begünstigen und inwiefern dabei organisationale Rahmenbedingungen eine Rolle spielen.

Zusammenfassend zeichnet sich für den Leiter A05 ein Führungsverständnis und Orientierungen des Führungshandelns ab, die sich maßgeblich an formalen bzw. formalisierten Regeln und an Merkmalen kooperativen Führens ausrichten. Durch seine Regelorientierung verleiht er den formalstrukturell implementierten Regeln subjektiv zusätzlich Geltung und befördert auf diese Weise zugleich die aufbauorganisatorisch bereits fest verankerte Koordinationsform Hierarchie. Sprichwörtlich im Schatten der Hierarchie scheint er durch die kooperativ ausgerichteten Bestandteile seines Führungsverständnisses zudem noch (mindestens) eine weitere Koordinationsform zu begünstigen: diejenige der Gemeinschaft. Indem er eine situativ geteilte Einflussnahme in Form von Team-Entscheidungen akzeptiert, durch die gemeinsame Entscheidungsfindung zwangsläufig die Binnenkommunikation erhöht, das WirErleben der Arbeitsgruppe stärkt und interne Unstimmigkeiten durch vermittelndes Verhandeln auszugleichen versucht, unterstützt er die Entwicklung von gemeinsamen Grundhaltungen und Gruppenkohäsion, die im Sinne der Governanceforschung gerade für Gemeinschaften so charakteristisch sind (vgl. Rosenstiel et al. 2005, S. 139; Kussau und Brüsemeister 2007, S. 41). Insbesondere sein kooperatives Führungsverständnis bzw. seine entsprechende Handlungsorientierung scheint mit Eigenschaften und Merkmalen der ÖFFENTLICH I in Verbindung zu stehen. Die relativ überschaubare Zahl von acht hauptberuflichen Mitarbeiterinnen erleichtert eine hohe Binnenkommunikation, die sich ablauforganisatorisch in ausgeprägten Gesprächsroutinen spiegelt und so förderliche Rahmenbedingungen für das Entstehen von sozialen Bindungen und gemeinsamen Überzeugungen hervorbringt. 
Demgegenüber beruht das Führungsverständnis und die damit verbundene Handlungsorientierung des Leiters A10 vorwiegend auf Grundsätzen delegativen Führens, die in Zielvereinbarungsgesprächen Umsetzung zu finden scheinen. Durch seine im Augenblick der Überprüfung des Vereinbarten demonstrierte Kontroll- und Sanktionsmacht reproduziert der Leiter A10 durch das Führungsinstrument ,Zielvereinbarungen“ einerseits die hierarchische Form der Handlungskoordination. Im Schatten der Hierarchie befördert A10 durch die Zielvereinbarungsgespräche andererseits zugleich eine Koordinationsform, die auf einem bedingten Belohnungssystem, auf wechselseitiger Beobachtung des Geleisteten sowie auf einer individuellen Unternehmerorientierung beruht, die sich mit den Analysemitteln der Governanceforschung als Koordination qua Wettbewerb beschreiben lässt (vgl. Benz und Dose 2010b, S. 258-259; Bonazzi 2008, S. 241). Ähnlich wie bei A05 lässt sich auch hier das primär delegative Führungsverständnis auf formalstrukturelle Merkmale der PRIVAT I zurückführen. Jedenfalls legen die Erzählungen des Leiters A10 nahe, dass die höhere Mitarbeiterzahl und mehr noch die durch Inhouse-Seminare bedingten geringen Präsenzzeiten seiner Mitarbeitenden eine Führungspraxis begünstigen, die ohne hohe Interaktionsdichte unter Anwesenden auskommt.

Insgesamt deutet der Fallvergleich darauf hin, dass Leitungspersonen in Weiterbildungsorganisationen durch ihr Führungsverständnis und ihre entsprechenden Handlungsorientierungen sowohl hierarchische als auch nicht-hierarchische Formen des koordinierten Zusammenwirkens ihrer Mitarbeitenden unterstützen. Auch wenn sie unterschiedliche Mittel hervorheben (Regeln vs. Zielvereinbarung), reproduzieren A05 und A10 in ihrem Führungsverständnis und ihren Handlungsorientierungen die formalstrukturell verankerte Hierarchie. Mit Gemeinschaft vs. Wettbewerb als nicht-hierarchischen Formen der Handlungsabstimmung scheinen empirische Variationen vor allem im Schatten der Hierarchie aufzufinden zu sein. Inwieweit hier personale und organisationale Merkmale konfundieren, lässt sich auf der Basis von Fallvergleichen nicht eindeutig entscheiden. Auf ihrer Grundlage können allerdings empirisch begründete Annahmen entwickelt werden, die Anknüpfungspunkte für weitere Forschung bieten. In diesem Sinne weist der hier dargestellte Fallvergleich darauf hin, dass eine gemeinschaftliche Koordination primär durch ein kooperatives Führungsverständnis und entsprechende Handlungsorientierungen unterstützt wird, die in einer Organisation mit geringer Mitarbeiterzahl und ausgeprägten formalisierten Gesprächsroutinen wahrscheinlicher zu sein scheinen. Demgegenüber wird eine wettbewerbliche Koordination primär durch ein delegatives Führungsverständnis und damit verbundene Handlungsorientierungen befördert, die sich eher unter den Bedingungen einer Organisation mit mittlerer Mitarbeiterzahl und geringer Interaktionsdichte unter Anwesenden auszubilden scheinen.

Inwiefern sich Variationen der organisationsinternen Führungs- und Koordinationspraxis auch auf die institutionelle Umwelt der Weiterbildungsorganisation zurückführen lassen, ist ein möglicher Anknüpfungspunkt für weitere Forschung. Im Anschluss an neoinstitutionalistische Überlegungen wäre zumindest denkbar, dass sich in Weiterbildungsorganisationen auch mit Blick auf die Führungs- und Koordinationspraxis institutionalisierte Rationalitätsvorstellungen ihrer relevanten Umwelt widerspiegeln (vgl. Meyer und Rowan 1977). Möglicherweise diffundieren manche Ausprägungen der Führungs- und Koordinationspraxis auch stärker als andere. So 
ließe sich etwa annehmen, dass der Leiter A05 sein regelgeleitetes, kooperatives Führungsverständnis und seine damit korrespondierenden Handlungsorientierungen auch in einer privatwirtschaftlichen Weiterbildungsorganisation realisieren könnte, wohingegen das maßgeblich auf Delegation durch bedingt honorierte Zielvereinbarungen beruhende Führungsverständnis bzw. die entsprechenden Handlungsorientierungen des Leiters A10 kaum in den Kontext Staat (vgl. Schrader 2011) überführbar zu sein scheinen - all dies wäre aber am empirischen Fall weiter zu klären.

Open Access Dieser Artikel wird unter der Creative Commons Namensnennung 4.0 International Lizenz (http://creativecommons.org/licenses/by/4.0/deed.de) veröffentlicht, welche die Nutzung, Vervielfältigung, Bearbeitung, Verbreitung und Wiedergabe in jeglichem Medium und Format erlaubt, sofern Sie den/die ursprünglichen Autor(en) und die Quelle ordnungsgemäß nennen, einen Link zur Creative Commons Lizenz beifügen und angeben, ob Änderungen vorgenommen wurden.

\section{Literatur}

Bäumer, J. (1999). Weiterbildungsmanagement: Eine empirische Analyse deutscher Unternehmen. München: Hampp.

Bea, F. X., \& Göbel, E. (2010). Organisation. Theorie und Gestaltung (4. Aufl.). Stuttgart: Lucius \& Lucius.

Benz, A., \& Dose, N. (2010a). Governance - Modebegriff oder nützliches sozialwissenschaftliches Konzept? In A. Benz \& N. Dose (Hrsg.), Governance - Regieren in komplexen Regelsystemen - Eine Einführung (2. Aufl. S. 13-36). Wiesbaden: VS.

Benz, A., \& Dose, N. (2010b). Von der Governance-Analyse zur Policytheorie. In A. Benz \& N. Dose (Hrsg.), Governance - Regieren in komplexen Regelsystemen - Eine Einführung (2. Aufl. S. 251-276). Wiesbaden: VS.

Blumenthal, J. (2005). Governance - Eine kritische Zwischenbilanz. Zeitschrift für Politikwissenschaft, 15(4), 1149-1180.

Blumer, H. (2004). Der methodologische Standort des symbolischen Interaktionismus. In J. Strübing \& B. Schnettler (Hrsg.), Methodologie interpretativer Sozialforschung. Klassische Grundlagentexte (S. 321-385). Konstanz: UTB.

Böhm, A., Legewie, H., \& Muhr, T. (2008). Kursus Textinterpretation: Grounded Theory. http://nbnresolving.de/urn:nbn:de:0168-ssoar-26629. Zugegriffen: 21. Januar 2016.

Bonazzi, G. (2008). Geschichte des organisatorischen Denkens. Wiesbaden: VS.

Brüsemeister, T. (2007). Analysemittel der Governanceforschung. In J. Kussau \& T. Brüsemeister (Hrsg.), Governance, Schule und Politik: Zwischen Antagonismus und Kooperation (S. 97-120). Wiesbaden: VS.

Crozier, M., \& Friedberg, E. (1979). Macht und Organisation - Die Zwänge kollektiven Handelns. Königstein: Athenäum.

Etzioni, A. (1975). A comparative analysis of complex organizations. On power, involvement, and their correlates. New York: Free Press.

Fombrum, C. (1984). Structures of organizational governance. Human Relations, 37(3), 207-234.

Froschauer, U., \& Lueger, M. (2003). Das qualitative Interview. Zur Praxis interpretativer Analyse sozialer Systeme. Wien: facultas.wuv.

Geulen, D. (1982). Soziales Handeln und Perspektivenübernahme. In D. Geulen (Hrsg.), Perspektivenübernahme und soziales Handeln: Texte zur sozial-kognitiven Entwicklung (S. 24-72). Frankfurt a. M.: Suhrkamp.

Glaser, B. G., \& Strauss, A. L. (2008). Grounded Theory. Strategien qualitativer Forschung. Bern: Huber.

Gnahs, D. (2010). Träger der Erwachsenenbildung. In R. Arnold, S. Nolda \& E. Nuissl (Hrsg.), Wörterbuch Erwachsenenbildung (2. Aufl. S. 288-289). Bad Heilbrunn: Klinkhardt.

Goulding, C. (2009). Grounded theory perspectives in organizational research. In D. Buchanan \& A. Bryman (Hrsg.), The SAGE handbook of organizational research methods (S. 381-394). London: SAGE.

Hentze, J., Graf, A., Kammel, A., \& Lindert, K. (2005). Personalführungslehre: Grundlagen, Funktionen und Modelle der Führung (4. Aufl.). Bern: UTB.

Herbrechter, D. (2016). Zur Bedeutung institutioneller Einflüsse für das Führungswissen in Weiterbildungsorganisationen. Hessische Blätter für Volksbildung, 66(3), im Druck. 
Hering, L., \& Schmidt, R. J. (2014). Einzelfallanalyse. In N. Baur \& J. Blasius (Hrsg.), Handbuch Methoden der empirischen Sozialforschung (S. 529-541). Wiesbaden: Springer VS.

Hopf, C. (1978). Die Pseudo-Exploration - Überlegungen zur Technik qualitativer Interviews in der Sozialforschung. Zeitschrift für Soziologie, 7(2), 97-115.

Kade, J., Nittel, D., \& Seitter, W. (2007). Einführung in die Erwachsenenbildung, Weiterbildung (2. Aufl.). Stuttgart: Kohlhammer.

Kelle, U. (2007). Qualitative Datenanalyse: computergestützt. Methodische Hintergründe und Beispiele aus der Forschungspraxis. In U. Kuckartz (Hrsg.), Theoretisches Vorwissen und Kategorienbildung in der "Grounded Theory" (2. Aufl. S. 32-49). Wiesbaden: VS.

Kelle, U., \& Kluge, S. (2010). Vom Einzelfall zum Typus. Fallvergleich und Fallkontrastierung in der qualitativen Sozialforschung (2. Aufl.). Wiesbaden: VS.

Kieser, A., \& Walgenbach, P. (2010). Organisation (6. Aufl.). Stuttgart: Schäffer-Poeschel.

Kuckartz, U., Dresing, T., Rädiker, S., \& Stefer, C. (2008). Qualitative Evaluation: Der Einstieg in die Praxis (2. Aufl.). Wiesbaden: VS.

Kussau, J., \& Brüsemeister, T. (2007). Educational Governance: Zur Analyse der Handlungskoordination im Mehrebenensystem der Schule. In H. Altrichter, T. Brüsemeister \& J. Wissinger (Hrsg.), Educational Governance. Handlungskoordination und Steuerung im Bildungssystem (S. 15-54). Wiesbaden: VS.

Lewin, K., Lippit, R., \& White, R. K. (1939). Patterns of aggressive behavior in experimentally created social climates. Journal of Social Psychology, 10, 271-299.

Locke, K. (2001). Grounded theory in management research. London: SAGE.

Lütz, S. (2010). Governance in der politischen Ökonomie I: Makro- und Mesoperspektiven. In A. Benz \& N. Dose (Hrsg.), Governance - Regieren in komplexen Regelsystemen - Eine Einführung (2. Aufl. S. 137-154). Wiesbaden: VS.

Martin, A., \& Langemeyer, I. (2014). Demografie, sozioökonomischer Status und Stand der Professionalisierung - das Personal in der Weiterbildung im Vergleich. In Deutsches Institut für Erwachsenenbildung (Hrsg.), Trends der Weiterbildung (S. 43-67). Bielefeld: wbv.

Mayntz, R., \& Scharpf, F.W. (1995). Steuerung und Selbstorganisation in staatsnahen Sektoren. In R. Mayntz \& F.W. Scharpf (Hrsg.), Gesellschaftliche Selbstregulierung und politische Steuerung (S. 9-38). Frankfurt a. M.: Campus.

Meyer, J., \& Rowan, B. (1977). Institutional organizations: formal structure as myth and ceremony. American Journal of Sociology, 83(2), 340-363.

Nerdinger, F. W. (2012). Grundlagen des Verhaltens in Organisationen (3. Aufl.). Stuttgart: Kohlhammer.

Neuberger, O. (2002). Führen und führen lassen: Ansätze, Ergebnisse und Kritik der Führungsforschung. Stuttgart: Lucius \& Lucius.

Peirce, C. S. (1991). Wie unsere Ideen zu klären sind. In C. S. Peirce \& K.-O. Apel (Hrsg.), Schriften zum Pragmatismus und Pragmatizismus (S. 182-214). Frankfurt a. M.: Suhrkamp.

Preedy, M., Bennett, N., \& Wise, C. (2012). Educational leadership. Context, strategy and collaboration. Los Angeles: SAGE.

Robak, S. (2004). Management in Weiterbildungsinstitutionen: Eine empirische Studie zum Leitungshandeln in differenten Konstellationen. Hamburg: Kovač.

Robak, S. (2012). Weiterbildungsmanagement. In B. Schäffer \& O. Dörner (Hrsg.), Handbuch Qualitative Erwachsenen- und Weiterbildungsforschung (S. 502-515). Opladen: Budrich.

Robak, S. (2015). Weiterbildungsmanagement. In J. Dinkelaker \& A. von Hippel (Hrsg.), Erwachsenenbildung in Grundbegriffen (S. 124-131). Stuttgart: Kohlhammer.

Rodler, C., \& Kirchler, E. (2002). Führung in Organisationen. Wien: WUV.

Rosenstiel, L. von, Molt, W., \& Rüttinger, B. (2005). Organisationspsychologie (9. Aufl.). Stuttgart: Kohlhammer.

Sauer-Schiffer, U. (2000). Biographie und Management: Eine qualitative Studie zum Leitungshandeln von Frauen in der Erwachsenenbildung. Münster: Waxmann.

Scharpf, F. W. (1991). Die Handlungsfähigkeit des Staates am Ende des 20. Jahrhunderts. Politische Vierteljahresschrift, 32(4), 621-634.

Schimank, U. (2010). Handeln und Strukturen: Einführung in die akteurtheoretische Soziologie (4. Aufl.). Weinheim: Juventa.

Schlüter, A. (2012). Leitungslernen - ein ungeplanter Lern- und Bildungsprozess? In C. Hof, J. Ludwig \& B. Schäffer (Hrsg.), Steuerung - Regulation - Gestaltung: Governance-Prozesse in der Erwachsenenbildung zwischen Struktur und Handlung (S. 154-164). Baltmannsweiler: Schneider Hohengehren.

Schlutz, E. (1997). Enttraditionalisierung, Modernisierung - oder ist die neue Weiterbildung noch die alte? Empirische Aufschlüsse aus der Bremer Untersuchung zum Weiterbildungsangebot. In K. De- 
richs-Kunstmann, P. Faulstich \& R. Tippelt (Hrsg.), Enttraditionalisierung der Erwachsenenbildung (S. 217-232). Frankfurt a. M.: DIE.

Schneider, V. (2004). Organizational Governance - Governance in Organisationen. In A. Benz \& N. Dose (Hrsg.), Governance - Regieren in komplexen Regelsystemen - Eine Einführung (S. 173-192). Wiesbaden: VS.

Schrader, J. (2011). Struktur und Wandel der Weiterbildung. Bielefeld: wbv.

Strauss, A. L. (1998). Grundlagen qualitativer Sozialforschung: Datenanalyse und Theoriebildung in der empirischen soziologischen Forschung (2. Aufl.). München: UTB. unveränderter Nachdruck

Strauss, A.L., \& Corbin, J. (1994). Grounded theory methodology: an overview. In N. K. Denzin \& Y. S. Lincoln (Hrsg.), Handbook of qualitative research (S. 273-285). Thousand Oaks: SAGE.

Strauss, A. L., \& Corbin, J. (1996). Grounded Theory: Grundlagen qualitativer Sozialforschung. Weinheim: Beltz.

Strübing, J. (2013). Qualitative Sozialforschung: Eine komprimierte Einführung für Studierende. München: Oldenbourg.

Tannenbaum, R., \& Schmidt, W.H. (1958). How to choose a leadership pattern. Harvard Business Review, $36(2), 95-101$.

Tietgens, H. (1994). Leiter/Leiterin und pädagogischer Mitarbeiter/pädagogische Mitarbeiterin an Volkshochschulen. Bielefeld: Bundesanstalt für Arbeit.

Truschkat, I., Kaiser-Belz, M., \& Volkmann, V. (2011). Theoretisches Sampling in Qualifikationsarbeiten: Die Grounded-Theorey-Methodologie zwischen Programmatik und Forschungspraxis. In G. Mey \& K. Mruck (Hrsg.), Grounded Theory Reader (2. Aufl. S. 353-380). Wiesbaden: VS.

Uhmann, C. (2011). Aktuelle Herausforderungen im Management von Weiterbildungsorganisationen. Hannover: Expressum.

Vroom, V.H., \& Jago, A. G. (1991). Flexible Führungsentscheidungen: Management der Partizipation in Organisationen. Stuttgart: Poeschel.

Weibler, J. (2012). Personalführung. München: Vahlen.

Weinert, A. B. (2004). Organisations- und Personalpsychologie (5. Aufl.). Weinheim: Beltz.

Wiesenthal, H. (2005). Markt, Organisation und Gemeinschaft als ,zweitbeste“ Verfahren sozialer Koordination. In W. Jäger \& U. Schimank (Hrsg.), Organisationsgesellschaft: Facetten und Perspektiven (S. 223-264). Wiesbaden: VS.

Williamson, O.E. (1985). The economic institutions of capitalism: firms, markets, relational contracting. New York: Free Press.

Wunderer, R. (2009). Führung und Zusammenarbeit: Eine unternehmerische Führungslehre (8. Aufl.). Köln: Luchterhand.

Yin, R. K. (2009). Case study research: design and methods (4. Aufl.). Thousand Oaks: SAGE. 